
Military Technical College Kobry El-Kobbah, Cairo, Egypt.

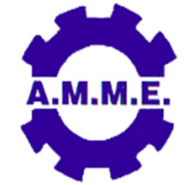

$18^{\text {th }}$ International Conference on Applied Mechanics and Mechanical Engineering.

\title{
LOW COST X-Y CORE POSITIONING SYSTEM USING STEPPER MOTOR
}

\author{
A. Y. AbdelHamid ${ }^{1, *}$, M. Abdeldayem ${ }^{1}$ and M. H. Mabrouk ${ }^{1}$
}

\begin{abstract}
$\mathrm{X}-\mathrm{Y}$ positioning is an important task in industrial applications, as this system is used for welding, cutting or for pick and place applications. The studied X-Y core positioning system is a parallel $X-Y$ position device, which due to less moved masses is potentially capable of fast acceleration and therefore faster positioning than traditional stacked systems. A kinematic model of $X-Y$ core positioning system is derived, which used for accurate position control in X-Y stage of the cart using low cost stepper motor and low cost controller (Arduino Mega2560). The proposed design of X-Y core positioning system which has 8 pulleys and two open ended derive-belts and have the double size of all designs that introduced in this field has its advantages of fast design, simplicity and flexibility. The system is very much simple, rugged, and cost effective. The experimental results show that the proposed system has a high resolution, repeatability and error is within acceptable limit. The proposed strategy used to control positioning in X-Y stage enable accurate cart positioning with max error percentage less than $5 \%$.
\end{abstract}

\section{KEYWORDS}

Belt-drive, xy core positioning system, Position Control, Stepper Motor.

Egyptian Armed Forces.

* Corresponding author; Email: ayahia@mtc.edu.eg. 


\section{INTRODUCTION}

X-Y positioning systems are widely used in industry to position a part or a tool within a two dimensional rectangular area. These systems are used for gantry, welding, cutting or for pick-and-place applications. Current implementations of X-Y positioning systems typically comprised of two linear guides, each of them has its own independent timing ball or belts /lead motors and screws [1,2]. One guide is stacked on top of the other, oriented perpendicularly to the other. Although the current configurations of $X-Y$ positioning systems are widely used, these systems tend to be bulky and they are not very suitable for low profile applications. High-speed systems are desirable in manufacturing because they can increase productivity. To provide a lightweight, low cost, compact positioning system, a single belt-driven H Frame Type $\mathrm{X}-\mathrm{Y}$ positioning system can be used as shown in Fig. 1. Such a system consists of two Guides that are parallel to each other, and a third guide vertical to the first two to form A shape.

A survey of the literature revealed that the first work describing a similar parallel-drive $\mathrm{X}-\mathrm{Y}$ positioning system as the developed $\mathrm{H}$-frame system was introduced by Sollmann [3]. The traditional stacked belt-drive systems or stacked ball-screw systems are the widely used $\mathrm{x}-\mathrm{y}$ positioning systems described. stacked ball screw $\mathrm{x}$ y positioning system control is described by H. Lim [4]. The paper considers a difference between motor position and end-effector position is caused by the finite stiffness of the ball screw. A torsional displacement feedback control was suggested for increasing the stalking in this work. In ref. [5], a positioning system comprising of two stacked belt-drive axis was studied. Hesitation in end-effector position is caused by using of elastic transmission elements (the belts). A sliding mode control strategy is used in both works to assign this problem. The above papers emphasize the need for an accurate model of the system to exist in order to effectively implement the control strategies. Elastic transmission and nonlinear friction are identified as the two major challenges in accurately controlling $X-Y$ positioning systems. or only one axis systems have also been carried out. Methods suggested to control one axis beltdrive systems described in literature include sliding mode control methods [6, 7], fuzzy-logic [8], PID control plus acceleration and friction compensation[9] , adaptive PID control [10], and feed-forward compensator under maximum acceleration and velocity constraints [11].

References [12-16] propose adaptive control strategies to address the problems caused by nonlinear friction in mechanical positioning systems. Yuki in [17] studied the motion mechanism of an X-Y micro-stage which designed and constructed for precision positioning of light Weight objects. A driving unit, which consists of two PZTs and a friction component made by permanent magnet, is mounted on the center of the stage base for driving the stage moving plate made by steel in the $X$ and $Y$-directions based on a friction drive. Again, most of those control strategies required the advance of an accurate model of the system to be controlled, which is the main emphasis in the present work.

From the literature, it was found that $\mathrm{H}$-bot's disadvantage is a racking of the gantry. The simply problem that Core $X-Y$ overpowers is the gantry rotating so if two situations were employed on an $\mathrm{H}$-bot. First, that the attached belt be attached and aligned on the center of the gantry's weight; Second, that the gantry be of such tolerance as to not rack even when trying to force it to do so by hand in rotational 


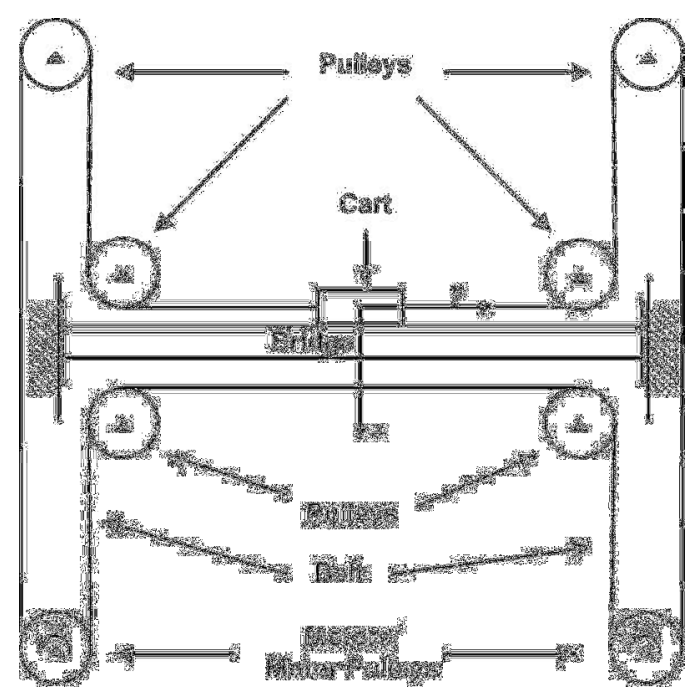

Fig. 1. General layout of the H-frame positioning system [3].

forces, but Core $X-Y$ doesn't prevent inertia or other forces from affecting the gantry. also the $\mathrm{H}$-bot's torque on the $y$-axis will add friction to the $y$-axis bearings even if the frame is stiff enough to not allow deformation. The friction could be eliminated by using good (expensive) bearings, but that more expensive than a few extra pulleys and a second belt. It is thought that each of the belts in the Core $X-Y$ are $75 \%$ of the length of the single belt in the H-bot. So you end up with more belt overall, but belt costs go up with continuous length, at some point while scaling up, the Core $X-Y$ becomes cheaper than the $\mathrm{H}$-bot. the longer spans of the $\mathrm{H}$-bot belt let for more stretching, sagging, etc. Core $X-Y$ is anti-racking [rotating] in many ways allowing for a looser/cheaper frame to be used while keeping quality and lightness. Finally, this paper is prepared as follows. In section II, the principle of operation of the proposed system is described. Section III presents the design and kinematic characteristics of the Core $X-Y$ positioning. Section IV presents the system configuration and experimental work. The results of the positioning proposed strategy is discussed in section $\mathrm{V}$. The concluding remarks are given in section $\mathrm{VI}$.

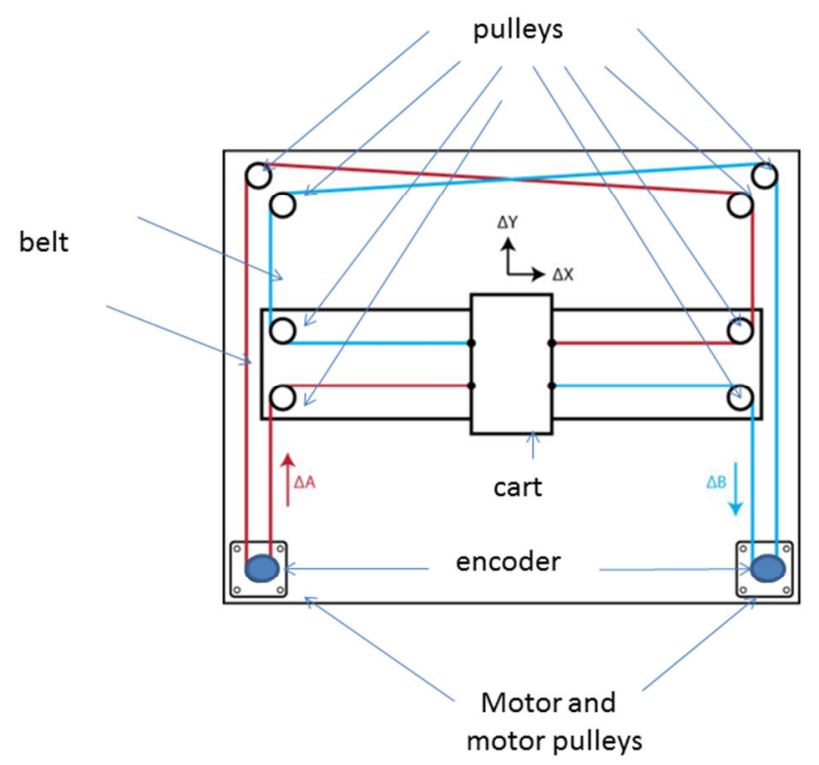

Fig. 2. General layout of the core XY positioning system. 


\section{PRINCIPAL OF OPERATION}

Direction of rotation setting is done by either reversing the connectors at the motors or controller board (only with power off or it may destroy the motor diver chip). or by inverting the directions logic via the firmware.

Here is how the mechanism works, paying no attention to any of the electrical stuff (driver pulleys rotation, motors at the front of mechanism): In Table 1 the effect of the rotation direction of each motor toward the cart direction is illustrated as follows:

The horizontal bar is a straight-edge which can be moved up and down by the user. This effect can be seen by following the direction of motion of the two cables which comprise the mechanism. Rotating both motors in the same direction results in horizontal motion as shown in Fig. 3 (A). Rotating both motors in opposite directions results in vertical motion as shown in Fig. 3 (B). If just one motor is rotate and the other still fixed the cart will move toward the diagonal direction. Rotating the alpha motor in $\mathrm{CCW}$ direction and fixing the beta motor results in diagonal motion toward the right-front direction as shown in Fig. 3 (C), while rotating the beta motor in CCW direction and fixing the alpha motor results in diagonal motion toward the right-rear direction as shown in Fig. 3 (D).

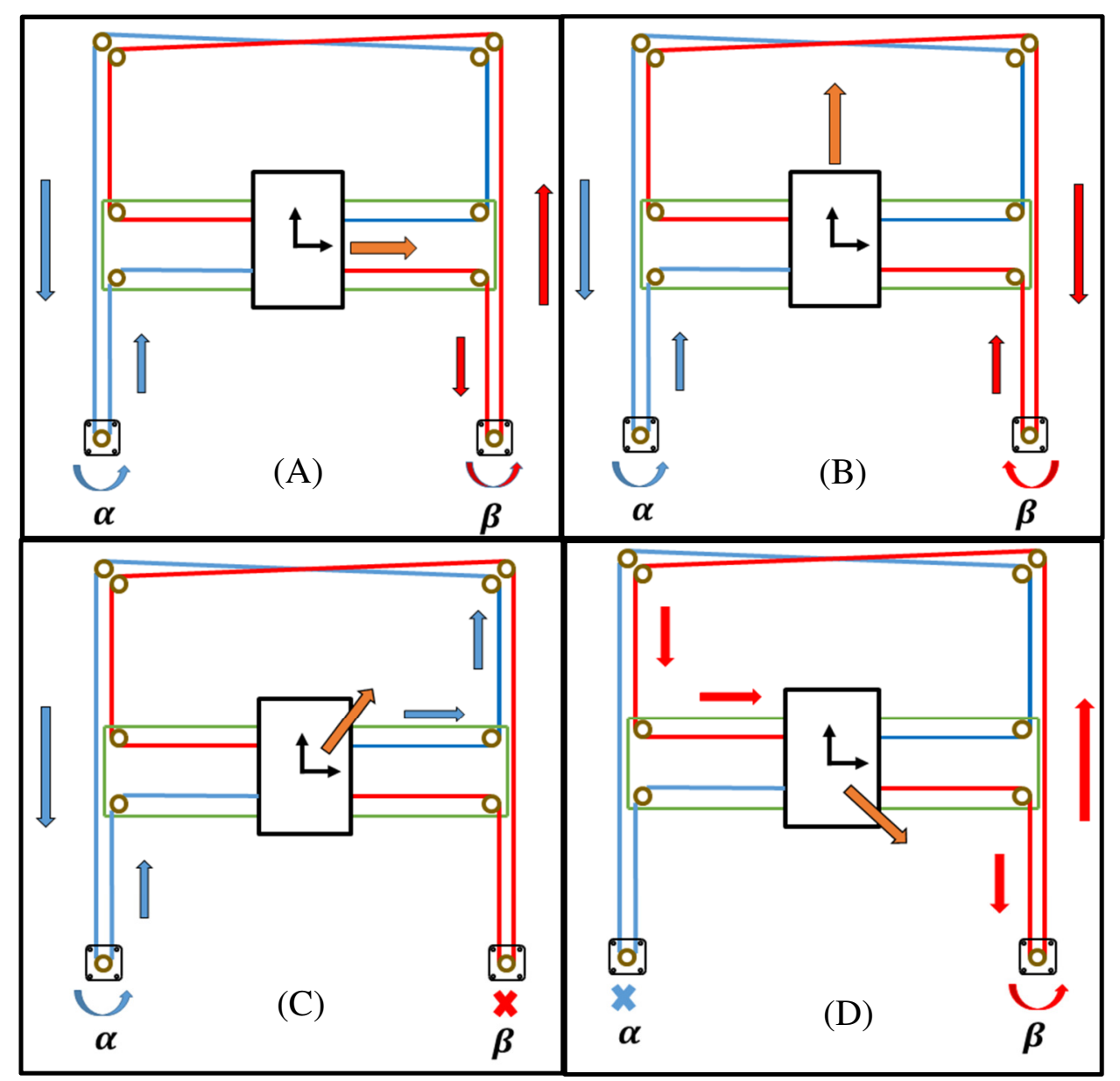

Fig. 3. X-Y Core positioning system configuration. 
Table 1. The direction of the cart according to motion of stepper motor.

\begin{tabular}{|c|c|c|}
\hline $\begin{array}{c}\text { Left Motor } \\
\text { (alpha) }\end{array}$ & $\begin{array}{c}\text { Right Motor } \\
\text { (beta) }\end{array}$ & Cart Motion \\
\hline cw & cw & left \\
\hline cw & ccw & rear \\
\hline ccw & cw & right \\
\hline ccw & ccw & left-front \\
\hline cw & off & right-rear \\
\hline ccw & off & left-rear \\
\hline off & cw & right-front \\
\hline off & ccw & \\
\hline
\end{tabular}

The location of the limit switches is important, when homing the mechanism, not the location of the origin. Homing should always direct the cart toward the switches. using the table above. just the top four entries

- Left and front, the alpha motor must turn $\mathrm{cw}$.

- Left and rear, the beta motor must turn $\mathrm{cw}$.

- Right and front, the beta motor turns ccw.

- Right and rear, the alpha motor turns ccw.

\section{DESIGN AND KINEMATIC MODELING}

In the $\mathrm{X}-\mathrm{Y}$ core positioning system shown in Fig. 2 there are two corresponding tracks along which a bridge is lead through on linear ball bearing-blocks. On the bridge there is a third track mounted, vertical to the first two tracks, on which a cart slides. Those three tracks form a X-Y core positioning system. On each end of the two parallel tracks sits one pulley, where the ones at the lower end are directly attached to the motor shaft. On each end of the track on the bridge, there are also two pulleys. Two open timing belt is guided around those eight pulleys including the motor pulleys. The open ends are both connected to the cart, which runs on the bridge. The system forms a parallel drive configuration, meaning that the actuator drive system is not an open kinematic chain. This parallel drive setup enables the rotational motion of the two stationary motors to transform into a linear $x$-motion of the bridge and a linear $y$-motion of the cart relative to the moving bridge. The overlapping of those two linear motions creates the xy-motion of the end effector. To relate the rotation of the two motors to the xy-motion of the cart, consider Fig. 2 again. Turning only one motor while keeping the other one still results in a linear motion of the end-effector in a $+/-45$ angle towards the $x y$-coordinate system. A positive rotation of motor 1 while holding motor 2 still results in a motion in negative $x$ and negative y directions, while a negative rotation of the same motor would cause a motion in positive $\mathrm{x}$ and positive $\mathrm{y}$ directions. Mathematically, this can be written as: 


$$
\begin{aligned}
\Delta X & =\frac{1}{2}(\Delta A+\Delta B) \\
\Delta Y & =\frac{1}{2}(\Delta A-\Delta B) \\
\Delta A & =\frac{1}{2}(\Delta X+\Delta Y) \\
\Delta A & =\Delta \alpha * r \\
\Delta B & =\frac{1}{2}(\Delta X-\Delta Y) \\
\Delta B & =\Delta \alpha * r
\end{aligned}
$$

From Eqn. (1) to Eqn. (6)

$$
\begin{aligned}
& \Delta X=\frac{1}{2}(\Delta \alpha * r+\Delta \beta * r) \\
& \Delta Y=\frac{1}{2}(\Delta \alpha * r-\Delta \beta * r) \\
& \Delta A=\Delta \alpha * r
\end{aligned}
$$

From Eqn. (3)

$$
r \Delta \alpha=-\Delta x-\Delta y
$$

where $\Delta \mathrm{x}, \Delta \mathrm{y}$, and $\Delta \boldsymbol{\alpha}$ is the change in $\mathrm{x}, \mathrm{y}$, and $\alpha$ direction, respectively; and $\mathrm{r}$ is the radius of the motor pulley. Note that the positive direction of rotation for each of the motors is defined as the mathematical positive rotation around the $z$-axis pointing outward from the paper plane, so the motor torques shown in Figure 1 are considered positive torques. Similarly, a positive rotation of motor 2 while holding motor 1 still would cause a motion in positive $x$ and negative $y$ directions, and a negative rotation of the same motor would cause a linear motion of the end-effector in negative $\mathrm{x}$ and positive y directions. Mathematically, this can be written as:

$r \Delta \beta=\Delta x-\Delta y$

where $\Delta \beta$ is the change in the $\beta$ direction. Solving equations (10) and (11) for $\Delta x$ and $\Delta y$, we get the following kinematic relationship between the axes:

$$
\left(\begin{array}{l}
\Delta x \\
\Delta y
\end{array}\right)=\left(\begin{array}{cc}
-\frac{1}{2} r & \frac{1}{2} r \\
-\frac{1}{2} r & -\frac{1}{2} r
\end{array}\right)\left(\begin{array}{l}
\Delta \alpha \\
\Delta \beta
\end{array}\right)
$$




\section{SYSTEM CONFIGURATION AND EXPERIMENTAL SETUP}

The configuration of the X-Y core positioning system shown in Fig. (4) is as follows: first the desired input target point is entered and the proposed trajectory planning is calculated online on the computer which give the order to the motor driver after converting the data to analog type and let the motor to move. To check that the motor tracks the desired path there is an incremental encoder which sense the rotation angles in type of pulse counter finally the computer calculates the error and give the correct action to let the motor keeps track the desired calculated path.

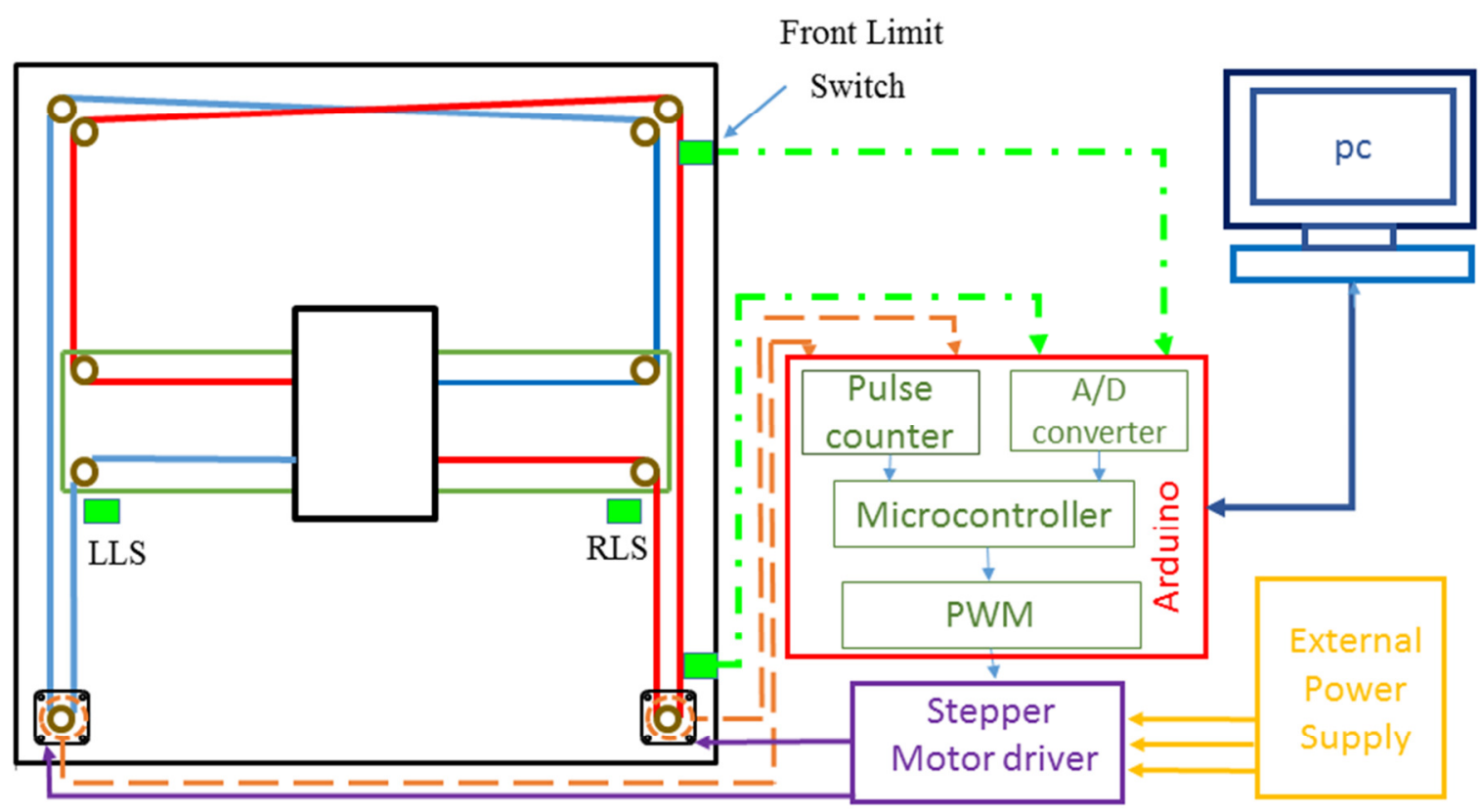

Fig. 4. $X-Y$ Core positioning system configuration.

The NEMA 23 stepper motor is connected to an easy driver motor controller board which is controlled by an Arduino Mega2560 microcontroller by means of wire connections as shown in block diagram of Fig.4. The Arduino program is uploaded in the microcontroller which controls the number of pulses to be generated for the stepper motor provided with a required amount of voltage level by means of easy driver circuit. Fig.4 shows the X-Y stage interfacing with PC.

The experimental prototype is shown in Fig. 5. The prototype is constructed of the base which is constructed of $16 \times$ V-Slot open builds Linear Rail $(40 \mathrm{~mm} \times 20 \mathrm{~mm} \times$ $1 \mathrm{~m})$ Black Anodized. The moving rack structure prototype is constructed of $1 \times \mathrm{V}$-Slot open builds Linear Rail $(40 \mathrm{~mm} \times 20 \mathrm{~mm} \times 1 \mathrm{~m})$ moving according to the base structure by means of. $V$ groove bearings linear motion directly without rails. The cart is made from a universal mounting platform which can use to mount another heavy moving axe, CNC router, 3D printer extruder or whatever heavy weight you want to carry and move. The footprint of the positioning system is $900 \mathrm{~mm}$ by $900 \mathrm{~mm}$ and the motion range is $810 \mathrm{~mm}$ along the $x$-axis and $810 \mathrm{~mm}$ along the $y$-axis. The motors used for the system are NEMA 23 Standard with holding torque of $9 \mathrm{Kg} . \mathrm{cm}$ and step angle (degrees) $1.8^{\circ}$. The timing belt type used is $2 \times$ GT2 Belt which has a $2 \mathrm{~mm}$ pitch, $6 \mathrm{~mm}$ width, open ended. The length of one belt is $3.95 \mathrm{~m}$. 


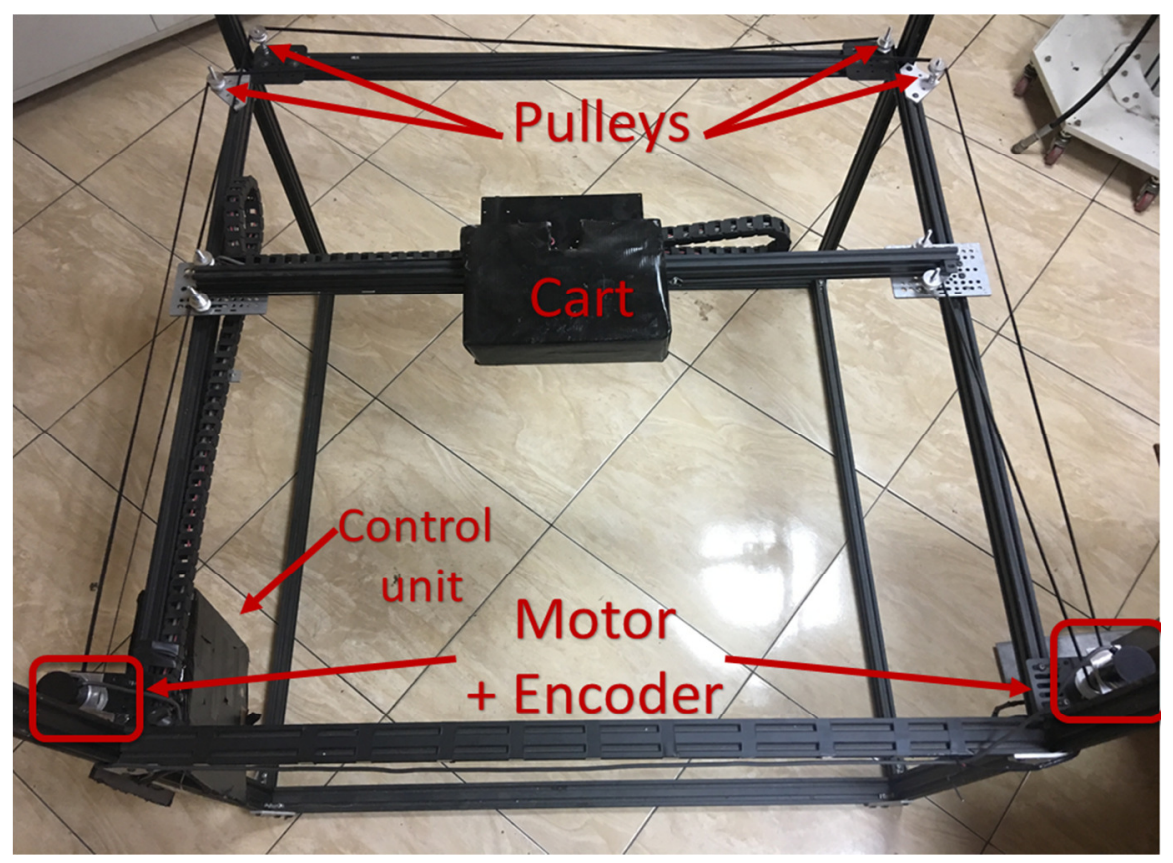

Fig. 5. Experimental work of $X-Y$ Core positioning system.

A sensor system shown in Fig.6 is constructed of two encoders A and B which are connected to each one of the stepper motors. These sensors measure the actual position of the cart along the $x$-axis and the $y$-axis The rotating angle of the stepper motor is measured using rotary encoder. The type of encoder is Omron quadrature incremental rotary encoder E6B2-CW6C with 1000 P/R (pulse per revolution), which has operating Voltage: 5 - 24VDC.

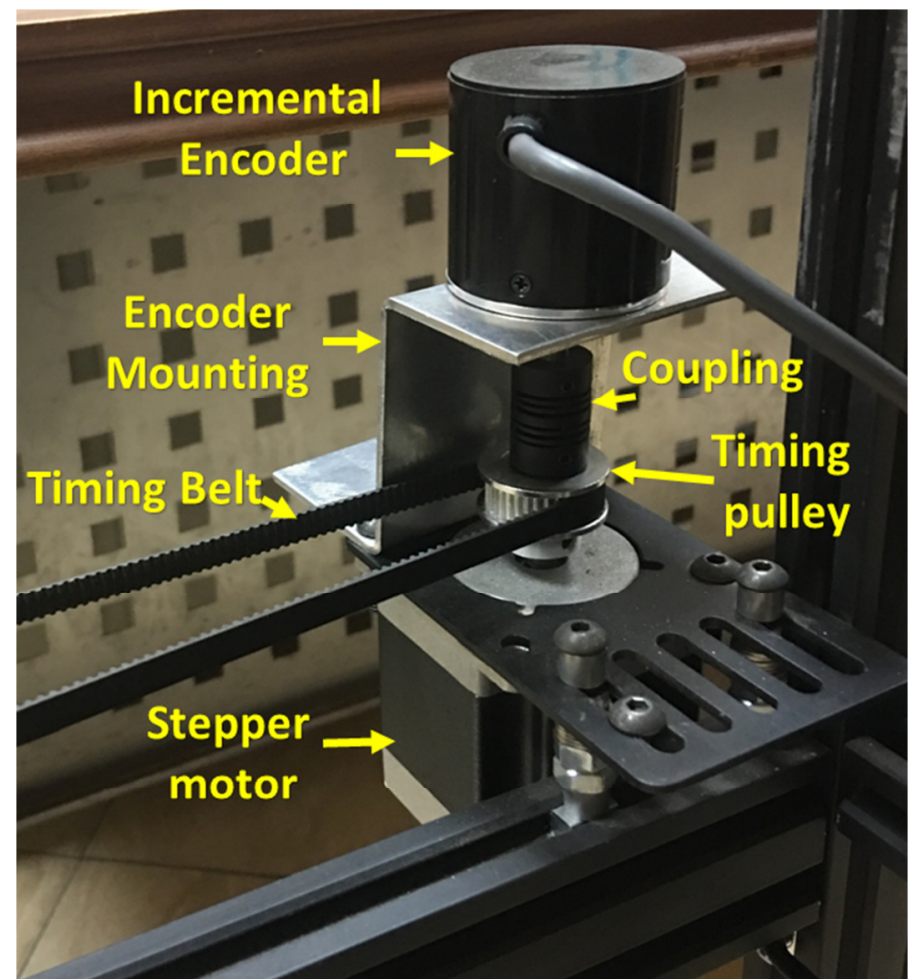

Fig. 6. X-Y Core sensor positioning system. 


\section{RESULTS AND DISCUSSION}

The experimental data is listed in Table 2 as follows:

Table 2. Experimental data for low cost core $x-y$ positioning system using stepper motor.

\begin{tabular}{|c|c|c|c|c|c|c|c|c|}
\hline \multirow{3}{*}{$\begin{array}{l}\text { Ser. } \\
\text { No. }\end{array}$} & \multicolumn{2}{|c|}{$\begin{array}{l}\text { Desired } \\
\text { target } \\
\mathrm{D}(\mathrm{mm})\end{array}$} & \multicolumn{2}{|c|}{$\begin{array}{l}\text { No.of steps to be } \\
\text { rotated by stepper } \\
\text { motor }\end{array}$} & \multirow{2}{*}{\multicolumn{2}{|c|}{$\begin{array}{l}\text { position achieved by the } \\
\text { stepper motor } \\
\text { corresponding to desired } \\
\text { position pressedin degree } \\
\text { Claculated }\left(x=n^{\star} 36 / 200\right)\end{array}$}} & \multicolumn{2}{|c|}{$\begin{array}{l}\text { Measured } \\
\text { Position from } \\
\text { encoder }\end{array}$} \\
\hline & \multirow{2}{*}{$X$} & \multirow{2}{*}{ Y } & \multirow{2}{*}{$\begin{array}{c}\text { Equiv. } \\
\text { Steps } \mathrm{n}= \\
X^{\star} 200 / \varphi_{p}\end{array}$} & \multirow{2}{*}{$\begin{array}{c}\text { Actual } \\
\text { stps }\end{array}$} & & & \multirow[b]{2}{*}{$X$} & \multirow[b]{2}{*}{$\mathrm{Y}$} \\
\hline & & & & & $\mathrm{X}$ & $\mathrm{Y}$ & & \\
\hline 1 & 10 & 10 & 55.55 & 56 & 10.08 & 10.1 & 10.1 & 9.8 \\
\hline 2 & 20 & 20 & 111.1 & 111 & 19.98 & 19.82 & 19.82 & 20.1 \\
\hline 3 & 30 & 30 & 166.66 & 167 & 30.06 & 29.9 & 29 & 30.06 \\
\hline 4 & 40 & 40 & 222.2 & 222 & 39.96 & 40.05 & 40.2 & 39.85 \\
\hline 5 & 50 & 50 & 277.7 & 278 & 50.04 & 50.1 & 49.7 & 50.54 \\
\hline 6 & 60 & 60 & 333.3 & 333 & 59.94 & $60 . .05$ & 60.5 & 59.2 \\
\hline 7 & 70 & 70 & 388.8 & 389 & 70.02 & 69.89 & 69.1 & 70.3 \\
\hline 8 & 80 & 80 & 444.4 & 444 & 79.92 & 79.85 & 78.5 & 79.95 \\
\hline 9 & 90 & 90 & 500 & 500 & 90 & 89.85 & 89.32 & 90.5 \\
\hline 10 & 100 & 100 & 555.55 & 556 & 100.08 & 100.12 & 101.1 & 100.02 \\
\hline 11 & 110 & 110 & 611.1 & 611 & 109.98 & 109.1 & 111.12 & 109.98 \\
\hline 12 & 120 & 120 & 666.6 & 667 & 120.06 & 120.15 & 121.12 & 120.06 \\
\hline
\end{tabular}

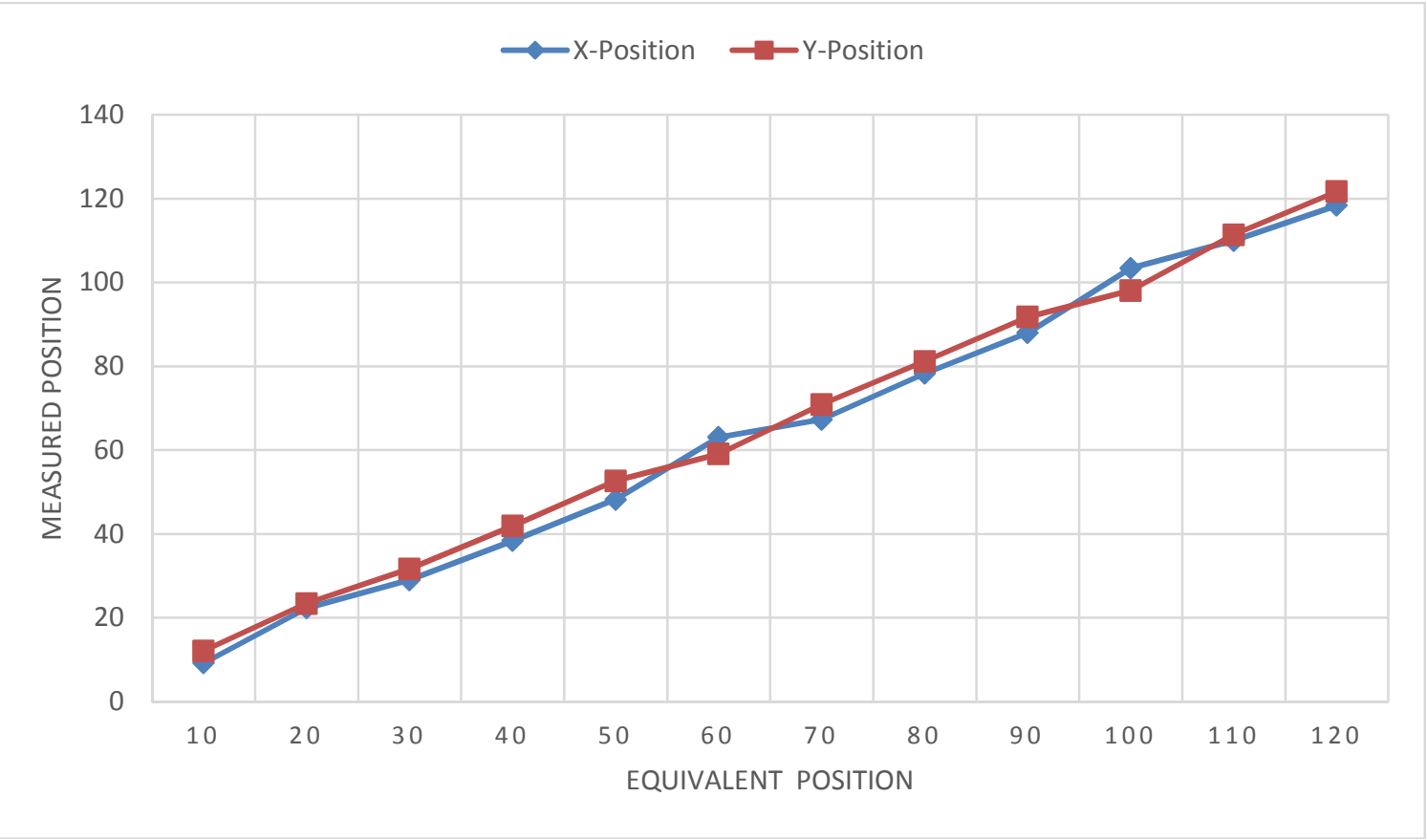

Fig. 7. Equivalent position Value ( $\mathrm{mm}$ ) from the Decoder vs. Measured Position (mm). 
Equivalent decimal value $(m)$ of the decoded output of the corresponding equivalent position has been plotted against the measured position in Fig.7. The curve shows a linear relationship. Calculated $X-Y$ position $(\mathrm{mm})$ and the measured $X-Y$ position $(\mathrm{mm})$, corresponding to the desired input $X-Y$ position plotted in Fig.8. This is also a linear curve. The percentage error in measuring the angular position has been shown in Fig.9 which indicates that the error lies within $\pm 3.77 \%$.

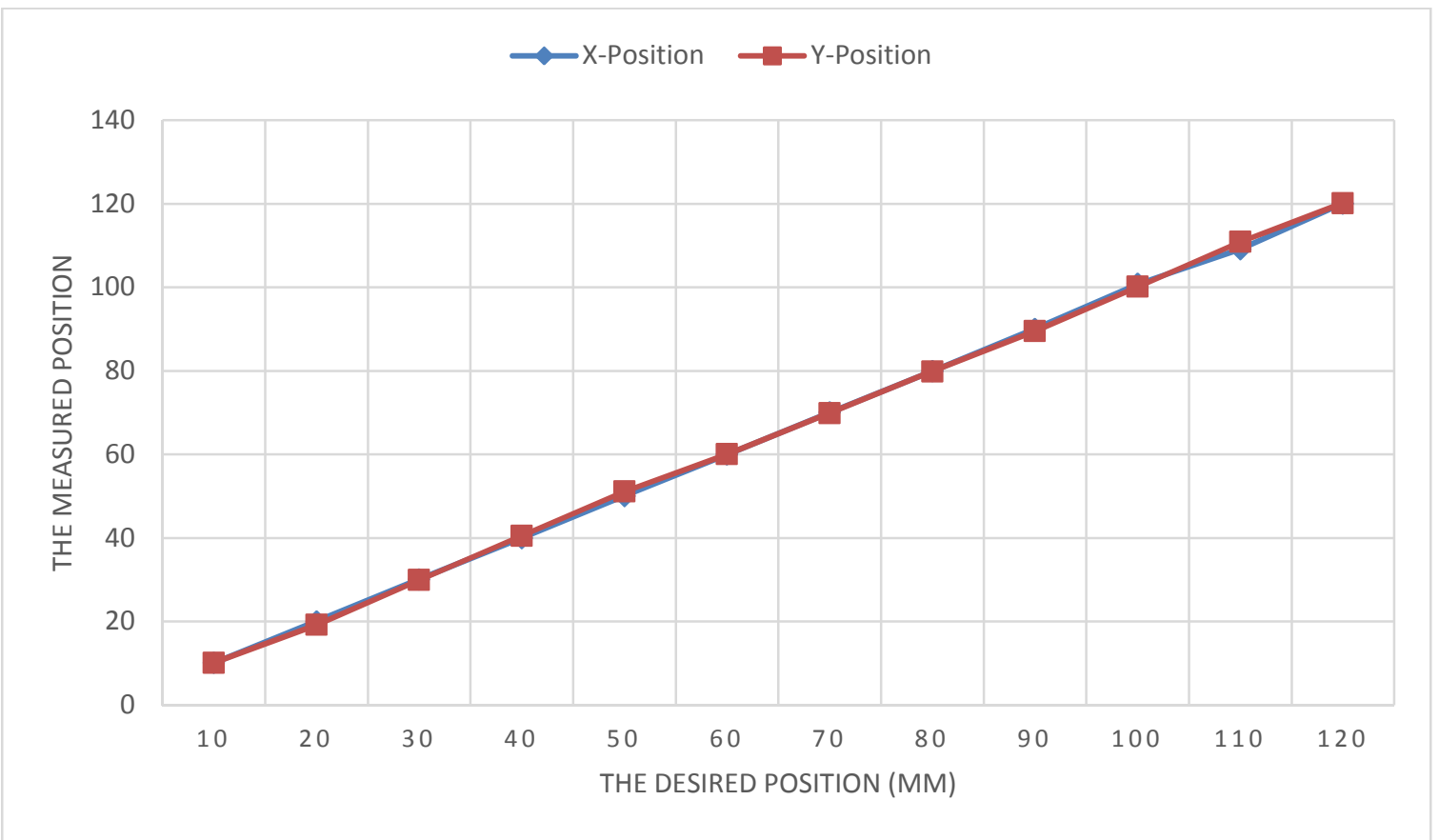

Fig. 8. Desired position Value (mm) vs. Measured Angular position (mm).

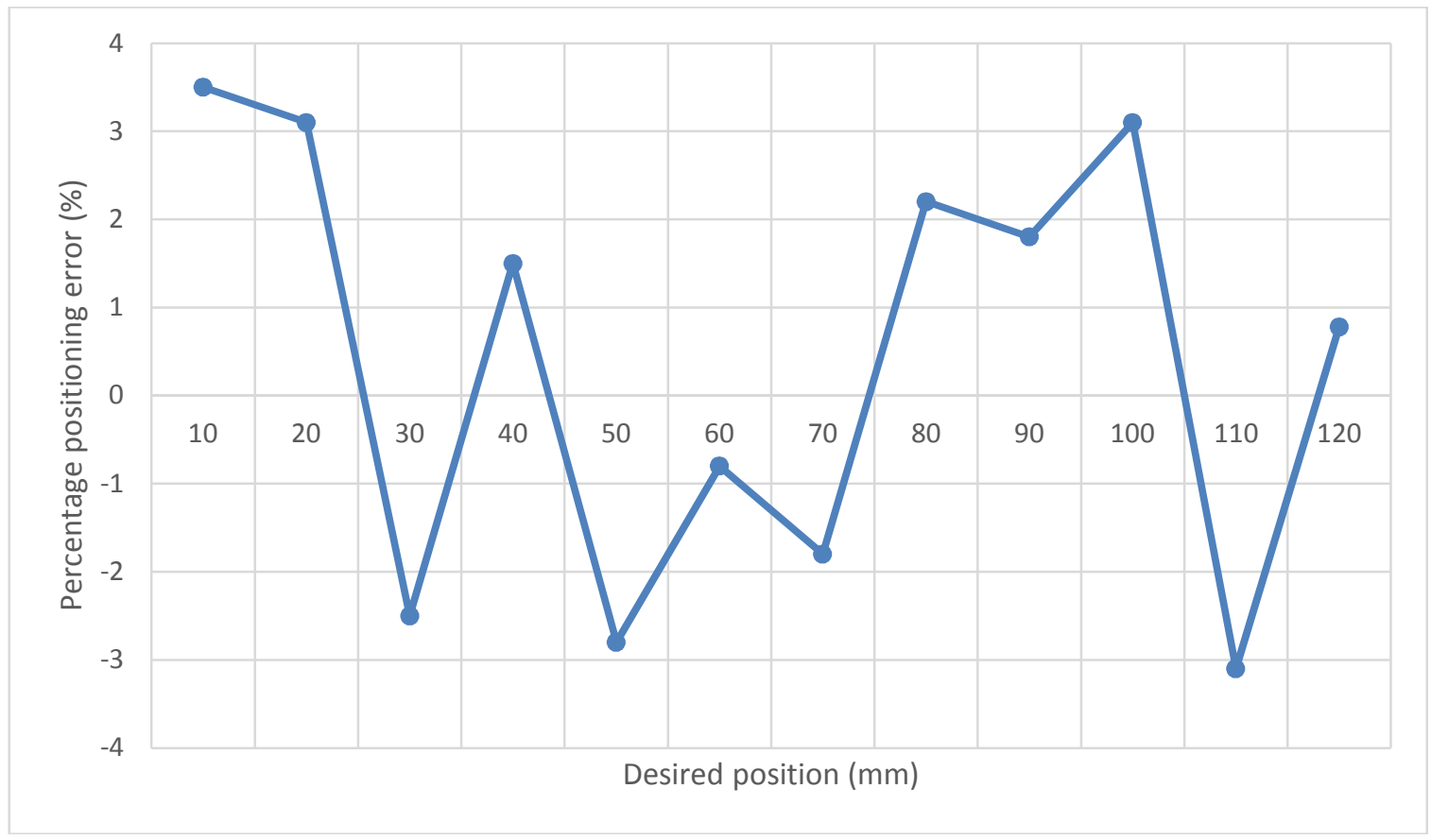

Fig. 9. Percentage error curve. 
From "Table 2", it is obtained that for serial no. 1-12 of the observation, the range of $X-Y$ position achieved is 10 to $120(\mathrm{~mm})$ with a resolution of 1 step angle $\left(1.8^{\circ}\right)$. If the desired angular position is beyond $120 \mathrm{~mm}$, the same way of calculation is done in the program at the microcontroller to achieve that desired position. As example, to achieve the X-Y position of $500 \mathrm{~mm}$, where $500 \mathrm{~mm}$ has equivalent steps of $\mathrm{n}=\left(200^{*} \mathrm{X}\right) / 36=2,777.7$ (stp), which mean the actual number of steps equal 2778 (stp). Thus any X-Y position in between 0 to $800(\mathrm{~mm})$ with a resolution of one step angle $\left(1.8^{\circ}\right)$ can be achieved. Also the mode of rotation i.e. forward or reverse can be selected by pressing the different mode of operation. The experimental results indicate that the system has good linearity and repeatability.

\section{CONCLUSIONS}

In this paper, a model for the dynamics of a belt driven, parallel-type, X-Y core positioning system was derived. The system developed in this paper is too simple, rough, and has cost effective. The experimental result displays that the model can accurately predict the response of the stage within the limited range of the sensors that were used. The model can be used in the design of closed-loop controllers to control the motion of the system.

The $X-Y$ Scanning Stage is micro-positioning stage which is driven by two bipolar stepper motors and controlled by Arduino Mega2560 microcontroller. This $X-Y$ Scanning Stage is designed and developed to precisely position the motion stage platforms along the predefined directions given by the special programs developed using Arduino software. Here, the two basic programs are developed to test the motion of the stages. The first program translates the motion stage in forward and backward motion at given delay time to reverse the direction. It uses the Easy Driver circuit and controls the single stage motor at a time. Second program is based on the Arduino shield board which controls the two motors one by one under a micro stepping mode with a speed control.

Experimentation is done to measure the $X$ and $Y$ motions with an incremental encoder at the speeds varying from 200 RPM to 1000 RPM and varying steps from 100 to 2000. The results obtained are within the acceptable limits. The accuracy within 38 microns is achieved. This work is beneficial in many micro-positioning and precision applications especially in 3D Printers. It also provides the cost effective way for system interpretation and electronics.

\section{REFERENCES}

[1] Y. Tomita, K. Makino, M. Sugimine, and N. Taniguchi, "High-response XY stage system driven by in-parallel linear motors," CIRP Annals-Manufacturing Technology, vol. 45, pp. 359-362, 1996.

[2] K. Itoh, M. Iwasaki, and N. Matsui, "Robust fast and precise positioning of ball screw-driven table system on machine stand," in Advanced Motion Control, 2004. AMC'04. The 8th IEEE International Workshop on, 2004, pp. 511-515.

[3] K. S. Sollmann, M. K. Jouaneh, and D. Lavender, "Dynamic modeling of a two-axis, parallel, H-frame-type XY positioning system," IEEE/Asme Transactions on Mechatronics, vol. 15, pp. 280-290, 2010. 
[4] H. Lim, J.-W. Seo, and C.-H. Choi, "Position control of XY table in CNC machining center with non-rigid ballscrew," in American Control Conference, 2000. Proceedings of the 2000,2000 , pp. 1542-1546.

[5] A. Hace, K. Jezernik, and M. Terbuc, "VSS motion control for a laser-cutting machine," Control Engineering Practice, vol. 9, pp. 67-77, 2001.

[6] A. Hace, K. Jezernik, and A. Sabanovic, "Improved design of VSS controller for a linear belt-driven servomechanism," IEEE/ASME Transactions on mechatronics, vol. 10, pp. 385-390, 2005.

[7] K. Jezernik and A. Sabanovic, "SMC with disturbance observer for a linear belt drive," IEEE Transactions on Industrial Electronics, vol. 54, pp. 3402-3412, 2007.

[8] A. S. Kulkarni and M. A. El-Sharkawi, "Intelligent precision position control of elastic drive systems," IEEE Transactions on Energy Conversion, vol. 16, pp. 26-31, 2001.

[9] W. Singhose, J. Vaughan, J. Danielson, and J. Lawrence, "Use of Cranes in System Dynamics and Controls Education," in World Congress, 2008, pp. 9099-9104.

[10] W. Li and X. Cheng, "Adaptive high-precision control of positioning tablestheory and experiments," IEEE Transactions on Control Systems Technology, vol. 2, pp. 265-270, 1994.

[11] T. Jayawardene, M. Nakamura, and S. Goto, "Accurate Control Position of Belt Drives under Acceleration and Velocity Constraints," International Journal of Control, Automation, and Systems, vol. 1, pp. 474-483, 2003.

[12] P. Vedagarbha, D. M. Dawson, and M. Feemster, "Tracking control of mechanical systems in the presence of nonlinear dynamic friction effects," IEEE Transactions on Control Systems Technology, vol. 7, pp. 446-456, 1999.

[13] M. Feemster, P. Vedagarbha, D. M. Dawson, and D. Haste, "Adaptive control techniques forfrictioncompensation," Mechatronics, vol. 9, pp. 125-145, 1999.

[14] Y. Hong and B. Yao, "A globally stable high-performance adaptive robust control algorithm with input saturation for precision motion control of linear motor drive systems," IEEE/ASME Transactions On Mechatronics, vol. 12, pp. 198-207, 2007.

[15] C.-I. Huang and L.-C. Fu, "Adaptive approach to motion controller of linear induction motor with friction compensation," IEEE/ASME transactions on mechatronics, vol. 12, pp. 480-490, 2007.

[16] C. Du, L. Xie, J. Zhang, and G. Guo, "Disturbance rejection for a data storage system via sensitivity loop shaping and adaptive nonlinear compensation," IEEE/ASME Transactions on Mechatronics, vol. 13, pp. 493-501, 2008.

[17] Y. Shimizu, Y. Peng, J. Kaneko, T. Azuma, S. Ito, W. Gao, et al., "Design and construction of the motion mechanism of an $X Y$ micro-stage for precision positioning," Sensors and Actuators A: Physical, vol. 201, pp. 395-406, 2013. 